\title{
The spread of a beneficial mutation in experimental bacterial populations: the influence of the environment and genotype on the fixation of rpos mutations
}

\author{
T Ferenci \\ School of Molecular and Microbial Biosciences, The University of Sydney, Sydney, New South Wales, Australia
}

\begin{abstract}
The spread of beneficial mutations through populations is at the core of evolutionary change. A long-standing hindrance to understanding mutational sweeps was that beneficial mutations have been slow to be identified, even in commonly studied experimental populations. The lack of information on what constitutes a beneficial mutation has led to many uncertainties about the frequency, fitness benefit and fixation of beneficial mutations. A more complete picture is currently emerging for a limited set of identified mutations in bacterial populations. In turn, this will allow quantitation of several features of mutational sweeps. Most importantly, the 'benefit'
\end{abstract}

of beneficial mutations can now be explained in terms of physiological function and how variations in the environment change the selectability of mutations. Here, the sweep of rpos mutations in Escherichia coli, in both experimental and natural populations, is described in detail. These studies reveal the subtleties of physiology and regulation that strongly influence the benefit of a mutation and explain differences in sweeps between strains and between various environments.

Heredity (2008) 100, 446-452; doi:10.1038/sj.hdy.6801077; published online 12 December 2007

Keywords: bacterial evolution; bacterial stress responses; continuous culture; chemostat culture; sigma factor $\sigma^{\mathrm{s}}$

\section{Introduction}

Spontaneous beneficial mutations contribute significantly to evolutionary change but remain one of the least understood aspects of biology (Orr, 1998; Hegreness et al., 2006). In the absence of fully characterized mutational sweeps, modelling mutational change was adopted in much of the early literature. Undoubtedly, the population biology approach and models of mutational takeovers were historically significant (Haldane, 1927; Fisher, 1930), but these and subsequent models necessarily needed to invoke assumptions on the fitness benefit conferred by mutations as well as their frequency in populations. The identity of beneficial mutations and the extent of the benefit have been elusive, although recent efforts have tried to estimate the distribution of benefits in experimental populations (Imhof and Schlotterer, 2001; Rozen et al., 2002; Barrett et al., 2006). This review focuses on a set of recently defined beneficial mutations arising in experimental continuous culture populations and what determines the conditional magnitude of their benefit under particular selection conditions.

With bacteria, beneficial sweeps should be readily studiable, but population changes called periodic

Correspondence: $\operatorname{Dr} T$ Ferenci, School of Molecular and Microbial Biosciences, The University of Sydney, G08, Sydney, New South Wales 2006, Australia.

E-mail: tferenci@mail.usyd.edu.au

Received 10 May 2007; revised 16 October 2007; accepted 28 October 2007; published online 12 December 2007 selection events were first observed indirectly through monitoring of the fluctuations in the proportion of selectively neutral mutations (Atwood et al., 1951). Until recently, with the exception of a set of mutations limited to the lac system (see references in Watt and Dean, 2000), the nature of beneficial mutations was not easy to study. The few previously characterized beneficial mutations involved regulatory changes in expression (Novick and Horiuchi, 1961; Adams, 2004). The difficulties in identifying beneficial mutations were discussed in a perceptive review a decade ago (Lenski et al., 1998) and even in well-studied bacteria like Escherichia coli, there is considerable difficulty in predicting what kind of change constitutes a beneficial mutation in a novel environment. Nevertheless, there has been progress in the past 10 years in the analysis of two kinds of experimental evolution system with E. coli cultures. In the Lenski long-term sub-cultured populations, several types of DNA change and fitness contributions have been discussed (Cooper et al., 2001; Lenski et al., 2003; Schneider and Lenski, 2004; Crozat et al., 2005; Pelosi et al., 2006). There has also been progress in identifying mutations in chemostats (continuous cultures) evolving in the presence of limiting glucose (Treves et al., 1998; Manche et al., 1999; NotleyMcRobb and Ferenci, 1999a, b, 2000; Notley-McRobb et al., 2002, 2003; Maharjan et al., 2006). Many of these examples deserve more detailed discussion, but in view of space limitations, this review is largely limited to one mutation that can be interpreted in terms of population sweeps, bacterial physiology and fitness effects under multiple growth conditions. This example illustrates 
many of the complex interplays between regulation, environment and mutation, which determine whether a mutation is beneficial. It also illustrates an example of trade-offs in evolution, long considered to be significant (MacArthur and Wilson, 1967), but for which detailed evidence has been scarce. In the case of rpos, the conditionality of the beneficial and detrimental effects can also be discussed.

\section{Strongly beneficial mutations in rpoS in E. coli populations growing under glucose limitation}

The rpos gene provides an excellent example of how counter-intuitive beneficial mutations can be. The $\sigma^{s}$ protein encoded by rpoS is the second most important sigma factor in E. coli and responsible for the expression of around $10 \%$ of E. coli genes, mostly contributing to the general stress response (Weber et al., 2005). Because of its high position in the hierarchy of transcriptional regulators, $\sigma^{\mathrm{S}}$ was expected to be conserved in varying environments. Yet in studying the expression of rpoS in glucose-limited chemostats, it was serendipitously observed that rpoS mutations occurred, and indeed spread at rapid rates within a few generations of establishing glucose-limited chemostats (Notley-McRobb et al., 2002). As shown in Figure 1, the selection coefficient of rpoS mutants is the highest of the regulatory mutations already identified in glucose-limited populations (Notley-McRobb et al., 2003). These mutations in rpoS, $m l c$, malT and $m g l D / O$ genes, all increased fitness and transport of limiting nutrient by distinct regulatory mechanisms (Notley-McRobb and Ferenci, 1999a,b). The $m l c, m g l$ and malT mutations resemble the earlier identified mutations and involve more limited regulatory changes in expression (Novick and Horiuchi, 1961).

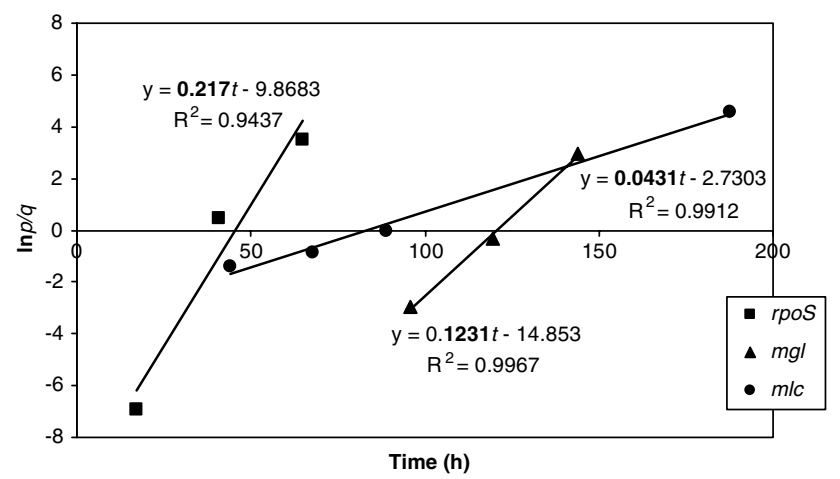

Figure 1 The selection coefficient of beneficial mutations spreading through E. coli populations. Bacteria inoculated into chemostats and subject to continuous glucose limitation accumulate mutations in several regulatory genes, including $r p o S, m g l D$ and $m l c$ (NotleyMcRobb et al., 2003). The spread of these mutations can be followed using simple phenotypic screens and the proportion of mutants relative to the rest of the population can be determined. With a dilution rate of $0.3 \mathrm{~h}^{-1}$ and a population size of $2 \times 10^{10}$ bacteria, the selection coefficient $s$ is determined by the slope of the linear regression of $\ln [p(t) / q(t)]$, where $p(t)$ and $q(t)$ represent the relative frequencies of the strains at times $t$ (Dykhuizen and Davies, 1980). The numbers in bold in the regression analyses indicate the selection coefficients ( $s$ ) for each sweep. The timescale is not based on a single culture; the data were obtained from the populations and sweeps described in Notley-McRobb and Ferenci $(2000,2002)$.
Consistent with the high selection coefficient of rpos sweeps, over $95 \%$ of the starting population is replaced in the cultures studied in Figure 1 within 3 days or 30 generations, growing at doubling times of $2.4 \mathrm{~h}$. The takeover is even quicker, within 10-12 generations, at slower growth rates. Also interesting is that the sequence of sweeps in glucose-limited cultures is inversely related to the selection coefficients of the three mutations in Figure 1. The sweep order $r p o S>m g l>m l c$ is reproducibly found in multiple replicate glucose-limited populations (Notley-McRobb et al., 2003). Whether selection coefficient always determine sweep order in evolving populations is unclear, but is clearly an important factor in large populations with no mutation supply problems. The decreasing selection coefficients in consecutive sweeps is also consistent with the finding that the fitness increase in evolving populations decreases asymptotically over extended periods of adaptation (Lenski et al., 1998).

\section{Nature of rpoS mutations in glucose-limited populations}

The majority of rpoS mutations accumulating in glucoselimited cultures are loss-of-function mutations with little or no residual RpoS protein. Sequencing of mutations from several populations has shown that sweeps such as in Figure 1 are due to concurrent enrichment of several distinct clones with distinct mutations (Notley-McRobb et al., 2002; unpublished data). The mutations include stop codons, deletions, insertions as well as point mutations. Given the population size of the chemostat cultures ( $>10^{10}$ bacteria), it is to be expected that multiple spontaneous loss-of-function mutations are present and all would have a similar fitness benefit. Indeed, phenotypic screens for rpoS function in chemostat-derived mutants demonstrate similar, drastic effects.

The replacement of the parental population by several independently arisen rpos mutants does have one important consequence. Superficially, the replacement of the chemostat population by the mutants is not diversifying in a phenotypic sense, but there are genetic implications. The separate genome lineages with different $r p o S$ mutations established in this first sweep lead to the possibility of other mutations differentially hitchhiking with the selected rpos mutations. This effect was most strikingly observed with mutator mutations that became prevalent in some chemostat populations by coselection with a subset of the sweeping mutants (NotleyMcRobb and Ferenci, 2000). Such events make seemingly straightforward periodic selections more complex and provide the source of later divergences in longer term populations. An example of this divergence was observed after 90 generations, when several rpoS alleles are stably established in chemostat populations, but with different mutational histories (Maharjan et al., 2006). The finding of divergence and the absence of purging periodic selections in experimental bacterial populations is not a new finding in itself and several explanations of diversification have been offered (Korona, 1996; Rainey and Travisano, 1998; Papadopoulos et al., 1999; MacLean and Bell, 2003). The rpoS findings add to explanations of diversity in populations. 
The loss of $\sigma^{\mathrm{S}}$ is not a lethal defect in unstressed cells, so in normal buffered media at optimal temperature and $\mathrm{pH}$, rpoS mutations do not cause a growth defect. However, when the culture medium is suboptimal or is subject to additional environmental stresses, the absence of stress resistance resulting from an rpoS null mutation becomes detrimental. Interestingly, the nature of the rpoS mutations in glucose-limited chemostats cultured at acidic $\mathrm{pH}$ (5.5) is altered, with a predominance of partial or attenuated mutations (Notley-McRobb et al., 2002). Such attenuated rpoS mutations, also observed in longterm stationary phase cultures (Zambrano et al., 1993), permit a reduced level of stress resistance but greater fitness in chemostats or stationary phase. The interesting antagonistic trade-offs that determine the benefit of rpoS mutations is considered below.

\section{The selectability of rpos mutations as a function of the environment and strain genotype}

The selection pressures on rpoS are largely due to the competition between the sigma factors $\operatorname{RpoS}\left(\sigma^{S}\right)$ and $\operatorname{RpoD}\left(\sigma^{D}\right)$ for a limiting number of RNA polymerase core subunits (Zhou and Gross, 1992; Farewell et al., 1998; Jishage and Ishihama, 1999). RpoS switches the cell to stress resistance whereas $\mathrm{RpoD}$ is needed for vegetative growth; an imbalance between the two can reduce the fitness of bacteria in particular situations. The hypothesis used to explain E. coli rpoS mutations in stationary phase batch cultures (Zambrano et al., 1993) or in steady-state glucose-limited chemostat populations (Notley-McRobb et al., 2002) is that mutations alleviate sigma factor competition and improve nutrient scavenging by increasing expression of RpoD-dependent genes (Ferenci, 2003). The trade-off in losing $\sigma^{\mathrm{s}}$ function is that rpoS mutants exhibit reduced resistance to stresses such as prolonged starvation, high pressure, high osmolarity, low $\mathrm{pH}$ as well as survival in the gastrointestinal tract (Cheville et al., 1996; Waterman and Small, 1996; Price et al., 2000; Dodd and Aldsworth, 2002; Bhagwat et al., 2006).

The antagonistic pleiotropy imposed by the sigma factor competition is one of several examples of the balancing of self-preservation and nutritional competence, or SPAN̄C balancing, in bacteria (Ferenci, 2005). rpoS mutations, being beneficial in some settings but not others, exhibit complex environment-by-genotype interactions that are amenable to study. The selection pressures on rpos are easiest to explain in schematic form as shown in Figure 2. Despite the overall complexity of the RpoS system, the rate at which rpoS mutations sweep populations in a particular environment can be reduced to a few quantifiable components. The first of these is the negative fitness effect on nutrient uptake, nutritional competence and vegetative growth resulting from high RpoS levels (Figure 2a). A second fitness input is the positive fitness contribution due to RpoS involvement in stress resistance in a particular environment. As found recently (King et al., 2006), the magnitude of both is sensitive to the particular surroundings. For example, at high osmolarity as shown as the stress in Figure 2a, the resistance due to RpoS is most important, and balances the nutritional advantage of elevated transport even under glucose limitation. In other more healthy environments, such as nutrient limitation at neutral $\mathrm{pH}$ (Figure 2a), the cost of RpoS-limiting transport outweighs the stress resistance benefit and rpos mutations readily sweep populations. The type of rpos allele enriched will also influence the magnitude of the costs and benefits in a sweep. When partial loss of function occurs, in some environments (Zambrano et al., 1993; Notley-McRobb et al., 2002), the fitness changes are less than with null mutants. The rate of rpoS mutation accumulation and type of mutation, as a function of the environment, have been estimated (King et al., 2006) and are discussed in the next section.

\section{Environmental determinants in the spread of rpos mutations}

The contribution of selection pressures to the magnitude of a sweep was measured in 15 controlled environments, including the eight shown in Figure 3 (King et al., 2006). We measured the rate of displacement of wild-type bacteria by rpoS mutants under nutrient limitation with and without additional stresses. As shown in Figure 3, there was considerable variation in the rate of sweeps. As described by the scheme in Figure 2, rpoS takeovers should occur particularly when RpoS has a strongly negative effect on fitness, that is when $\sigma^{s}$ levels are high but when a stress response is not needed for viability. In Figure 2a, this combination of events is shown for nutrient-limited bacteria in an otherwise unstressed environment.

The estimation of the negative effect was carried out with bacteria in a chemostat culture by measuring the transport of limiting nutrient. In chemostats, transport rate at limiting substrate concentrations is related to fitness (Novick and Szilard, 1950; Hansen and Hubbell, 1980; Notley-McRobb and Ferenci, 1999a). The rpoS influence is measurable from a comparison of glucose uptake rates in bacteria with and without intact rpoS, comparing isogenic strains differing only in rpos. The inactivation of RpoS and removal of sigma factor competition indeed increase expression of all genes involved in glucose uptake, which are RpoD-dependent (Notley-McRobb et al., 2002; Seeto et al., 2004). By measuring glucose transport in each of the modified environments in rpoS and $r p o S^{+}$bacteria, the repressive effect can be revealed and compared to the magnitude of the selection pressure for loss of rpoS in experimental sweeps.

As shown in Figure 3, environments such as anaerobiosis prevent or greatly reduce the spread of rpoS mutations when compared to aerobic glucose-limited chemostats at identical growth rates. At the same time, the environments still provide a selection pressure for the loss of RpoS, since a transport difference between rpoS and $r p o S^{+}$bacteria was measurable in all cases. The observation that rpoS mutants did not spread in proportion to the transport difference is consistent with the prediction in Figure 2 that a fitness effect provided by stress resistance also contributes to the magnitude of the rpoS sweep. A reservation at this point is that we have no evidence that the transport difference is linearly related to fitness and this needs detailed analysis in the future. Nevertheless, in the case of anaerobiosis, low tempera- 
a

a Rate of sweep by

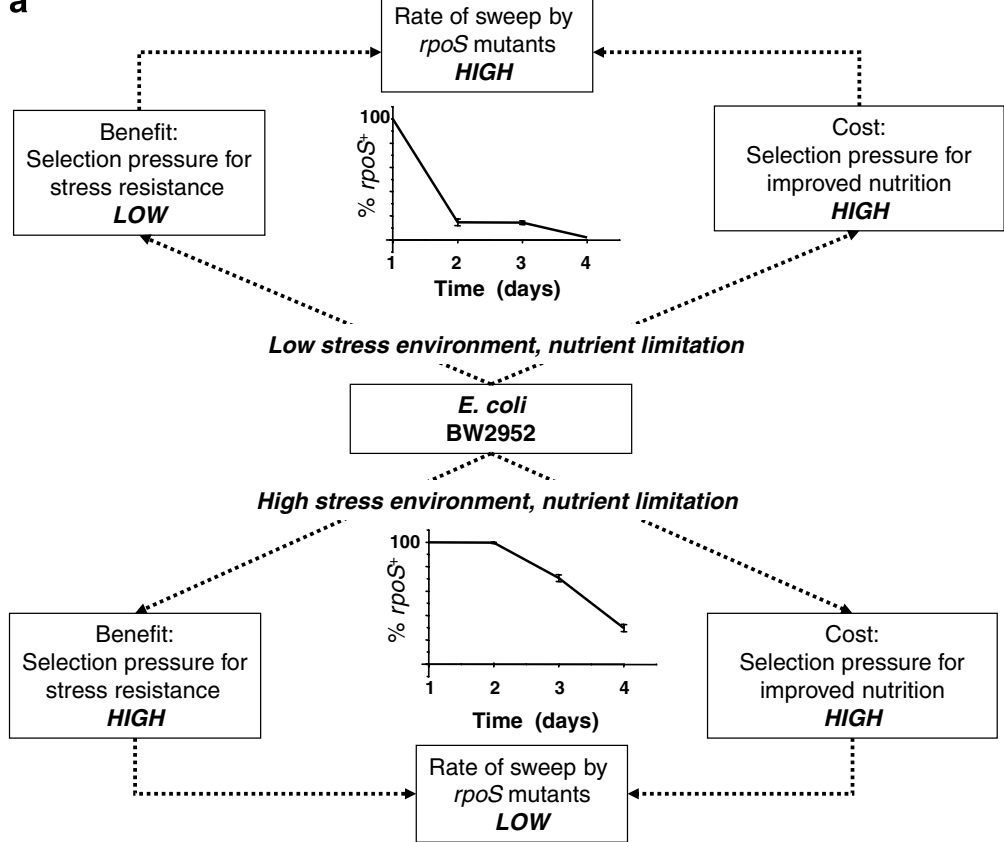

b Rate of sweep by rpos mutants

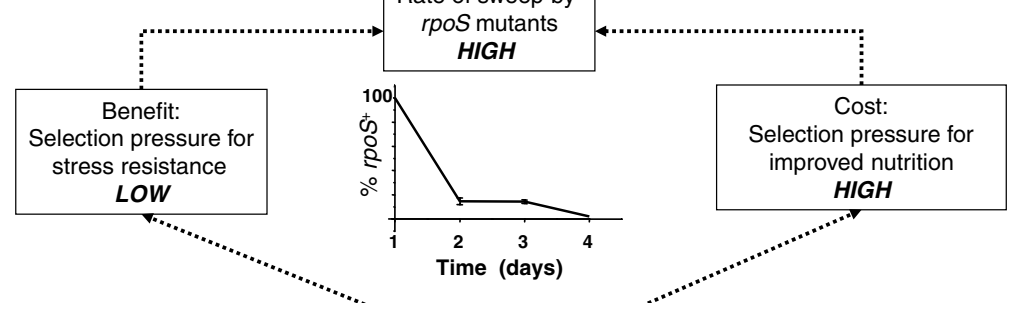

Low stress environment, nutrient limitation

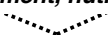

BW2952 (HIGH endogenous RpoS level)

MG1655 (LOW endogenous RpoS level) ............

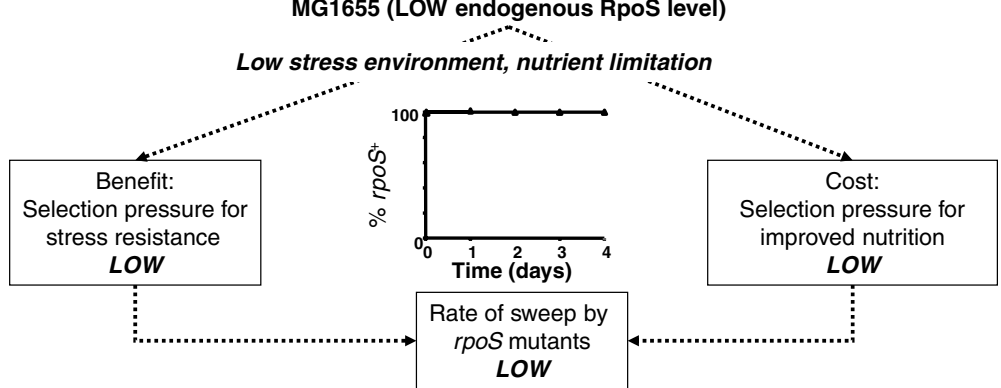

Figure 2 Major influences affecting the selectability of rpoS mutations in E. coli. The spread of rpoS mutations in E. coli populations is affected by the environment (a) and the genotype of the strain (b). (a) The environment determines the level of cellular $\sigma^{\mathrm{s}}$ through multiple input signals (Weber et al., 2005), and is higher under stress conditions. A particular concentration of $\sigma^{\mathrm{s}}$ is postulated to have two effects on fitness. First, $\sigma^{\mathrm{S}}$ contributes to viability by inducing the general stress response and provides a fitness contribution that is zero in unstressed bacteria (e.g. at $37^{\circ} \mathrm{C}, \mathrm{pH} \mathrm{7,} \mathrm{no} \mathrm{osmotic} \mathrm{stress,} \mathrm{no} \mathrm{oxidative} \mathrm{stress,} \mathrm{etc.)} \mathrm{but} \mathrm{positive} \mathrm{under} \mathrm{suboptimal} \mathrm{conditions} \mathrm{(e.g.} \mathrm{at} 25^{\circ} \mathrm{C}$, $\mathrm{pH} 5.5$, high osmolarity etc.). In (a) the high-stress environment inset is with high osmolarity (King et al., 2006). In unstressed bacteria, a high level of $\sigma^{\mathrm{s}}$ has a negative effect on fitness because it competes with $\sigma^{\mathrm{D}}$ for available RNA polymerase and reduces expression of vegetative genes dependent on $\sigma^{\mathrm{D}}$. The competition results in lower expression of functions, like transport, essential for growth. The transport difference between $r p o S^{+}$and $r p o S^{-}$ bacteria in a particular environment is indicative of this negative fitness effect (Figure 3). Especially with low-nutrient environments (either limitation for good substrates like glucose or maltose, or growth with poor carbon sources like acetate that give low growth rates), rpo $S$ expression is elevated in otherwise unstressed bacteria. The negative transport effect is high under these conditions (see Figure 3 below), so results in high rates of $r p o S$ sweep and provides a selection pressure for the rpoS mutation (see insets for the pattern of rpoS sweeps in each condition; the data on glucose-limited chemostats with and without osmotic stress is from King et al., 2006). (b) Different strains of E. coli (both lab strains and natural isolates) vary in their $\sigma^{\mathrm{S}}$ content (King et al., 2004; Bhagwat et al., 2006). The genotype of $E$. coli determines $\sigma^{\mathrm{S}}$ levels, as the concentration of $\sigma^{\text {s }}$ under stress conditions varies considerably between strains (Ferenci, 2005). Strains with genotypedetermined high levels of RpoS protein (such as BW2952) have a greater cost (in terms of transport effect), even more under selection for $r p o S$ mutations. MG1655 on the other hand has lower endogenous $\sigma^{\mathrm{S}}$ and the selection pressure for rpoS mutation is reduced. The inset $r p o S$ sweep data is from King et al. (2004). 


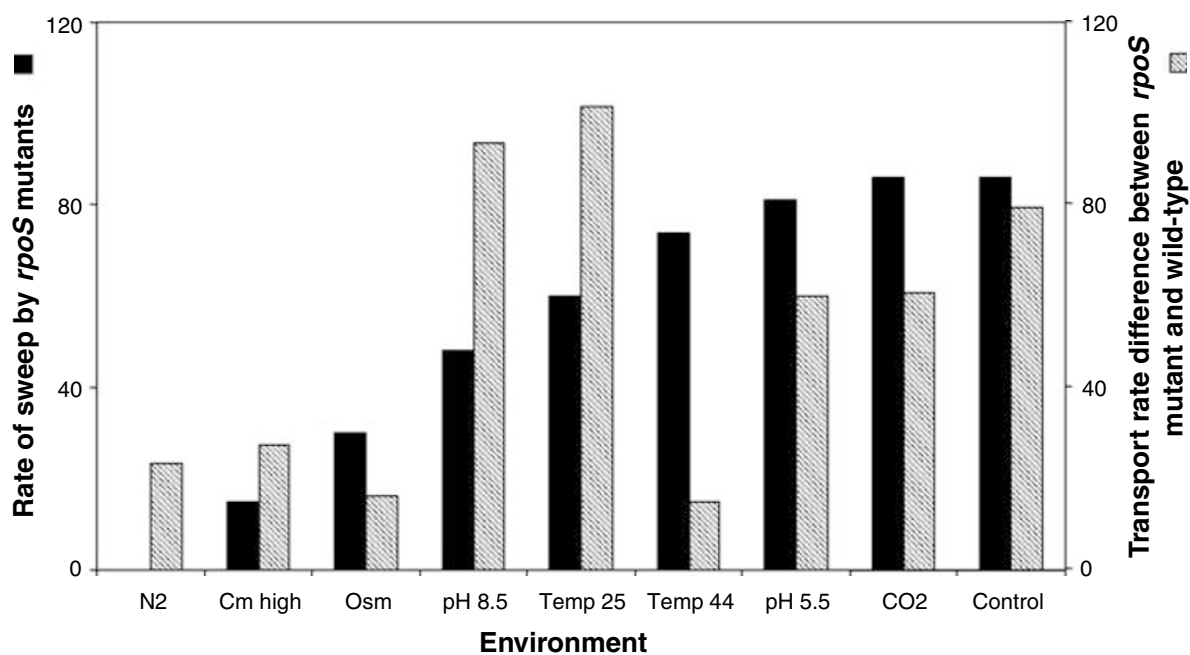

Figure 3 Estimation of the rate of sweeps and the selection pressure for beneficial mutations in rpoS. The 'control' in Figure 3 represents the cultures of $E$. coli grown in glucose-limited chemostats at $37^{\circ} \mathrm{C}, \mathrm{pH} 7$, no osmotic stress, with aeration and at $D=0.1 \mathrm{~h}^{-1}$. This environment was modified by changing the medium or atmosphere in eight different ways indicated in Figure 3 and described in more detail in King et al. (2006). Briefly, $\mathrm{CO}_{2}$ represents a culture atmosphere in which $\mathrm{N}_{2}$ was replaced by $\mathrm{CO}_{2} ; \mathrm{Temp}$ or $\mathrm{pH}$ indicates a changed culture temperature or $\mathrm{pH}$ respectively; Osm indicates elevated osmolarity of the medium; $\mathrm{Cm}$ high indicates growth in the presence of sublethal chloramphenicol concentrations; $\mathrm{N}_{2}$ represents an anaerobic environment. In each environment, the magnitude of the sweep was obtained from measuring the increasing proportion of rpoS bacteria over the first 4 days of culture. A high value is indicative of rapid sweeps. The transport rate difference between $\mathrm{rpoS}^{+}$and $\mathrm{rpoS}^{-}$bacteria was determined directly under each growth condition as also described in King et al. (2006).

ture or high $\mathrm{pH}$, the selection of rpoS mutants was much lower than could be explained by the considerable magnitude of the transport cost. Other findings are also consistent with the significance of $\sigma^{\mathrm{s}}$ in particular environments such as under anaerobiosis (King and Ferenci, 2005). Although additional factors other than those considered in Figure 2 may contribute, the simplified scheme in Figure 2 will provide a means of analysing, for the first time, the physiological factors influencing a mutational sweep.

The study of rpos sweeps illustrates the exquisite sensitivity of the fitness benefit to environmental conditions. The conditionality of fitness effects is even more dramatically demonstrated by malT mutations in chemostat cultures. These mutations switch from being beneficial to detrimental simply by changing the growth rate. Under glucose limitation, malT mutations are beneficial and selected at $D=0.1 \mathrm{~h}^{-1}$, near-neutral at $0.3 \mathrm{~h}^{-1}$ but are detrimental at $0.6 \mathrm{~h}^{-1}$ (Notley-McRobb et al., 2003). Selectability of mutations is that sensitive to changes in bacterial physiology.

\section{Strain polymorphisms and the influence of genotype on the spread of rpos mutations}

One of the several unexpected findings on the spread of rpoS mutations was that the speed and extent of the sweep shown in Figure 1 was remarkably strain-specific in different $E$. coli strains. As shown in Figure $2 \mathrm{~b}$, the benefit conferred by rpoS mutations under identical conditions of glucose limitation showed considerable strain variation. In some strains, the rpoS fitness benefit in chemostats is even greater than with BW2952, the strain generally used in my lab as the ancestor in evolution experiments. In other strains such as the often-used MG1655, the rpoS mutations are undetectable in glucose-limited cultures at times when BW2952 populations are already >95\% rpoS (King et al., 2004 and Figure $2 \mathrm{~b}$ ). Consequently, the host genotype is as important as the environment in determining the benefit of the rpos mutation.

The underlying difference between strains is in the control of the endogenous level of $\sigma^{\mathrm{s}}$ protein in the cell. Under identical growth conditions, strains vary as much as 10 -fold in $\sigma^{\mathrm{S}}$ protein per cell (King et al., 2004). High endogenous RpoS results in higher sigma factor competition against RpoD, and E. coli strains with high $\sigma^{\mathrm{S}}$ levels indeed accumulated rpos mutations at a higher rate during growth under nutrient limitation, relieving the competition. The high $-\sigma^{S}$ strains have a reduced fitness in any environment where nutrition is the selective determinant. Disruption of rpoS indeed improves nutritional capability not just under glucose limitation but also with many poor carbon sources of $E$. coli like acetate or succinate (Chen et al., 2004; King et al., 2004).

The genetic basis underlying differences in $\sigma^{\mathrm{s}}$ levels is unknown, although intragenic polymorphisms undoubtedly contribute (Atlung et al., 2002). Indeed, the widespread occurrence of polymorphisms in and near rpoS indicates that the selection pressures on the SPANC balance described in Figure 2 occur in natural environments (Ferenci, 2003). Consequently, changes in the SPANC trade-off in nature results in heterogeneity in several phenotypes associated with stress resistance (Bhagwat et al., 2006). Many inputs control $\sigma^{\text {s }}$ levels at the transcriptional and post-transcriptional phases of rpoS expression, so changes in numerous genes could influence protein levels and stress resistance. One recently identified contributor is linked to the spoT region present in a strain (Spira and Ferenci, 2008). spoT, also involved in global gene regulation, may also be expected to be subject to the same SPANC trade-offs as rpoS (Spira and Ferenci, 2008). No doubt other extragenic polymorphisms also explain $\sigma^{\mathrm{s}}$ level heterogeneity. 


\section{The fixation of rpos mutations}

Despite the magnitude of the fitness advantage conferred by $r p o S$ mutations in high-RpoS strains under glucose limitation, none of the eight populations studied so far became $100 \%$ rpos. The proportion of $\mathrm{rpoS}^{+}$bacteria decreased to below $1 \%$ in some populations, but the $\mathrm{rpoS}^{+}$sub-population was stably maintained. Indeed, in all chemostat cultures studied, the proportion of rpoS ${ }^{+}$ bacteria recovered within 100 generations to become $>30 \%$ of the population (Maharjan et al., 2006). An important question arising from these findings is if such very strongly beneficial mutations as in rpoS do not lead to complete fixation, is it likely that any mutation in a large bacterial population purges diversity? The everincreasing complexity of long-term populations almost to the level of individuality (Papadopoulos et al., 1999; Maharjan et al., 2007) suggests a negative answer to this question. The universality of this conclusion will of course depend on data with other cell types and experimental systems.

The co-existence of multiple rpoS genotypes and the co-existence of both rpoS ${ }^{+}$and $r p o S$ sub-populations are the likely sources of divergence in chemostat populations (Maharjan et al., 2006). More weakly beneficial mutations than those in Figure 1 have almost no chance of leading to fixation, and the slow turnover of sub-populations over several weeks of further adaptation in chemostats suggests that purging periodic events are not a feature of continually adapting bacteria. Beyond the first 50 generations under glucose limitation, the mutations with high selection coefficients are supplemented by numerous other mutations affecting multiple phenotypic characteristics such as regulation, metabolism, transport and outer membrane permeability (Maharjan et al., 2006). Because of space considerations, the diversifying effects of mutations with low selection coefficients will not be described here. Nevertheless, the incomplete fixation of mutations and the co-existence of mutations was a previously underestimated aspect of bacterial population change, but is also being observed in populations other than chemostat cultures (de Visser and Rozen, 2006). A possible basis of diversification is frequency-dependent selection (Friesen et al., 2004) and this is the explanation used to explain diversification in a more complex mixture of resources (Barrett and Bell, 2006). In our chemostat isolates, however, frequency-dependent selection was tested but does not explain the diversity in evolving chemostat populations (Maharjan et al., 2006, 2007).

\section{Conclusions and prospects}

The 'benefit' in beneficial mutations is a function of the interplay between genotype, environment and the physiology of a cell. In the case of rpoS mutations, the selectivity and speed of mutational spread is determined by the quantifiable trade-off costs in a particular environmental setting. The sensitivity of the benefit, the incomplete fixation of strongly beneficial mutations and the demonstrated divergence associated with weakly beneficial mutations are inconsistent with simple models of bacterial evolution. A simple black-box approach to bacterial properties in population models is no longer justifiable.
Up to now, the rpoS sweep represents one of the few beneficial mutations that have been amenable to detailed analysis. In the future, the identification and investigation of beneficial mutations will be easier and facilitated by the application of genomics. In the past few months, microbial genomes of lab-evolved organisms have been sequenced and mutations identified (Herring et al., 2006; Velicer et al., 2006). The complete cataloguing of all the mutations removes the earlier restrictions of trying to guess what beneficial mutations arise as well as the constraint of our incomplete understanding of organisms.

\section{Acknowledgements}

I thank the Australian Research Council for funding support.

\section{References}

Adams J (2004). Microbial evolution in laboratory environments. Res Microbiol 155: 311-318.

Atlung T, Nielsen HV, Hansen FG (2002). Characterisation of the allelic variation in the rpoS gene in thirteen $\mathrm{K} 12$ and six other non-pathogenic Escherichia coli strains. Mol Genet Genomics 266: 873-881.

Atwood KC, Schneider LK, Ryan FJ (1951). Selective mechanisms in bacteria. Cold Spring Harb Symp Quant Biol 16: 345-355.

Barrett RDH, Bell G (2006). The dynamics of diversification in evolving Pseudomonas populations. Evolution 60: 484-490.

Barrett RDH, M'Gonigle LK, Otto SP (2006). The distribution of beneficial mutant effects under strong selection. Genetics 174: 2071-2079.

Bhagwat AA, Tan J, Sharma M, Kothary M, Low S, Tall BD et al. (2006). Functional heterogeneity of RpoS in stress tolerance of enterohemorrhagic Escherichia coli strains. Appl Environ Microbiol 72: 4978-4986.

Chen GZ, Patten CL, Schellhorn HE (2004). Positive selection for loss of RpoS function in Escherichia coli. Mutat Res 554: 193-203.

Cheville AM, Arnold KW, Buchrieser C, Cheng CM, Kaspar CW (1996). RpoS regulation of acid, heat, and salt tolerance in Escherichia coli O157-H7. Appl Environ Microbiol 62: 1822-1824.

Cooper VS, Schneider D, Blot M, Lenski RE (2001). Mechanisms causing rapid and parallel losses of ribose catabolism in evolving populations of Escherichia coli B. J Bacteriol 183: 2834-2841.

Crozat E, Philippe N, Lenski RE, Geiselmann J, Schneider D (2005). Long-term experimental evolution in Escherichia coli. XII. DNA topology as a key target of selection. Genetics 169: 523-532.

de Visser JAGM, Rozen DE (2006). Clonal interference and the periodic selection of new beneficial mutations in Escherichia coli. Genetics 172: 2093-2100.

Dodd CER, Aldsworth TG (2002). The importance of RpoS in the survival of bacteria through food processing. Int J Food Microbiol 74: 189-194.

Dykhuizen D, Davies M (1980). An experimental model: bacterial specialists and generalists competing in chemostats. Ecology 61: 1213-1227.

Farewell A, Kvint K, Nystrom T (1998). Negative regulation by RpoS-a case of sigma factor competition. Mol Microbiol 29: 1039-1051.

Ferenci T (2003). What is driving the acquisition of mutS and rpoS polymorphisms in Escherichia coli? Trends Microbiol 11: 457-461.

Ferenci T (2005). Maintaining a healthy SPANC balance through regulatory and mutational adaptation. Mol Microbiol 57: 1-8. 
Fisher RA (1930). The Genetical Theory of Natural Selection. OUP: Oxford.

Friesen ML, Saxer G, Travisano M, Doebeli M (2004). Experimental evidence for sympatric ecological diversification due to frequency-dependent competition in Escherichia coli. Evolution 58: 245-260.

Haldane JBS (1927). The mathematical thoery of natural and artificial selection, part V: selection and mutation. Proc Camb Philos Soc 23: 838-844.

Hansen SR, Hubbell SP (1980). Single-nutrient microbial competition: qualitative agreement between experimental and theoretically forecast outcomes. Science 207: 1491-1493.

Hegreness M, Shoresh N, Hartl D, Kishony R (2006). An equivalence principle for the incorporation of favorable mutations in asexual populations. Science 311: 1615-1617.

Herring CD, Raghunathan A, Honisch C, Patel T, Applebee MK, Joyce AR et al. (2006). Comparative genome sequencing of Escherichia coli allows observation of bacterial evolution on a laboratory timescale. Nat Genet 38: 1406-1412.

Imhof M, Schlotterer C (2001). Fitness effects of advantageous mutations in evolving Escherichia coli populations. Proc Natl Acad Sci USA 98: 1113-1117.

Jishage M, Ishihama A (1999). Transcriptional organization and in vivo role of the Escherichia coli rsd gene, encoding the regulator of RNA polymerase sigma D. J Bacteriol 181: 3768-3776.

King T, Ferenci T (2005). Divergent roles of RpoS in Escherichia coli under aerobic and anaerobic conditions. FEMS Microbiol Lett 244: 323-327.

King T, Ishihama A, Kori A, Ferenci T (2004). A regulatory trade-off as a source of strain variation in the species Escherichia coli. J Bacteriol 186: 5614-5620.

King T, Seeto S, Ferenci T (2006). Genotype-by-environment interactions influencing the emergence of $r p o S$ mutations in Escherichia coli populations. Genetics 172: 2071-2079.

Korona R (1996). Genetic divergence and fitness convergence under uniform selection in experimental populations of bacteria. Genetics 143: 637-644.

Lenski RE, Mongold JA, Sniegowski PD, Travisano M, Vasi F, Gerrish PJ et al. (1998). Evolution of competitive fitness in experimental populations of E. coli-what makes one genotype a better competitor than another. Antonie Van Leeuwenhoek 73: 35-47.

Lenski RE, Winkworth CL, Riley MA (2003). Rates of DNA sequence evolution in experimental populations of Escherichia coli during 20,000 generations. J Mol Evol 56: 498-508.

MacArthur RH, Wilson EO (1967). The Theory of Island Biogeography. Princeton University Press: Princeton, New Jersey, USA.

MacLean RC, Bell G (2003). Divergent evolution during an experimental adaptive radiation. Proc $R$ Soc Lond B Biol Sci 270: $1645-1650$.

Maharjan R, Seeto S, Ferenci T (2007). Divergence and redundancy of transport and metabolic rate-yield strategies in a single Escherichia coli population. J Bacteriol 189: 2350-2358.

Maharjan R, Seeto S, Notley-McRobb L, Ferenci T (2006). Clonal adaptive radiation in a constant environment. Science 313: 514-517.

Manche K, Notley-McRobb L, Ferenci T (1999). Mutational adaptation of Escherichia coli to glucose limitation involves distinct evolutionary pathways in aerobic and oxygenlimited environments. Genetics 153: 5-12.

Notley-McRobb L, Ferenci T (1999a). Adaptive $m g l$-regulatory mutations and genetic diversity evolving in glucose-limited Escherichia coli populations. Environ Microbiol 1: 33-43.

Notley-McRobb L, Ferenci T (1999b). The generation of multiple coexisting mal-regulatory mutations through polygenic evolution in glucose-limited populations of Escherichia coli. Environ Microbiol 1: 45-52.
Notley-McRobb L, Ferenci T (2000). Experimental analysis of molecular events during mutational periodic selections in bacterial evolution. Genetics 156: 1493-1501.

Notley-McRobb L, King T, Ferenci T (2002). rpoS mutations and loss of general stress resistance in Escherichia coli populations as a consequence of conflict between competing stress responses. J Bacteriol 184: 806-811.

Notley-McRobb L, Seeto S, Ferenci T (2003). The influence of cellular physiology on the initiation of mutational pathways in Escherichia coli populations. Proc R Soc Lond B Biol Sci 270: 843-848.

Novick A, Horiuchi T (1961). Hyper-production of betagalactosidase by Escherichia coli bacteria. Cold Spring Harb Symp Quant Biol 26: 239-245.

Novick A, Szilard L (1950). Experiments with the chemostat on spontaneous mutations of bacteria. Proc Natl Acad Sci USA 36: 708-719.

Orr HA (1998). The population genetics of adaptation-the distribution of factors fixed during adaptive evolution. Evolution 52: 935-949.

Papadopoulos D, Schneider D, Meier-Eiss J, Arber W, Lenski RE, Blot M (1999). Genomic evolution during a 10,000generation experiment with bacteria. Proc Natl Acad Sci USA 96: 3807-3812.

Pelosi L, Kuhn L, Guetta D, Garin J, Geiselmann J, Lenski RE et al. (2006). Parallel changes in global protein profiles during long-term experimental evolution in Escherichia coli. Genetics 173: 1851-1869.

Price SB, Cheng CM, Kaspar CW, Wright JC, Degraves FJ, Penfound TA et al. (2000). Role of $r p o S$ in acid resistance and fecal shedding of Escherichia coli O157: H7. Appl Environ Microbiol 66: 632-637.

Rainey PB, Travisano M (1998). Adaptive radiation in a heterogeneous environment. Nature 394: 69-72.

Rozen DE, de Visser J, Gerrish PJ (2002). Fitness effects of fixed beneficial mutations in microbial populations. Curr Biol 12: 1040-1045.

Schneider D, Lenski RE (2004). Dynamics of insertion sequence elements during experimental evolution of bacteria. Res Microbiol 155: 319-327.

Seeto S, Notley-McRobb L, Ferenci T (2004). The multifactorial influences of RpoS, Mlc and cAMP on $p t s G$ expression under glucose-limited and anaerobic conditions. Res Microbiol 155: 211-215.

Spira B, Ferenci T (2008). Alkaline phosphatase as a reporter of $\sigma^{\mathrm{s}}$ levels and rpoS polymorphisms in different $E$. coli strains. Arch Microbiol 189: 43-47.

Treves DS, Manning S, Adams J (1998). Repeated evolution of an acetate-crossfeeding polymorphism in long-term populations of Escherichia coli. Mol Biol Evol 15: 789-797.

Velicer GJ, Raddatz G, Keller H, Deiss S, Lanz C, Dinkelacker I et al. (2006). Comprehensive mutation identification in an evolved bacterial cooperator and its cheating ancestor. Proc Natl Acad Sci USA 103: 8107-8112.

Waterman SR, Small PL (1996). Characterization of the acid resistance phenotype and rpoS alleles of shiga-like toxinproducing Escherichia coli. Infect Immun 64: 2808-2811.

Watt WB, Dean AM (2000). Molecular-functional studies of adaptive genetic variation in prokaryotes and eukaryotes. Annu Rev Genet 34: 593-622.

Weber H, Polen T, Heuveling J, Wendisch VF, Hengge R (2005). Genome-wide analysis of the general stress response network in Escherichia coli: sigma(S)-dependent genes, promoters, and sigma factor selectivity. J Bacteriol 187: 1591-1603.

Zambrano MM, Siegele DA, Almiron M, Tormo A, Kolter R (1993). Microbial competition: Escherichia coli mutants that take over stationary phase cultures. Science 259: 1757-1760.

Zhou Y, Gross C (1992). How a mutation in the gene encoding sigma 70 suppresses the defective heat shock response caused by a mutation in the gene encoding sigma 32 . J Bacteriol 174: 7128-7137. 